\title{
COMPARISON OF A MODIFIED SELF-ORgANIZING MigRATING ALGORITHM WITH OTHER SELECTED OPTIMIZATION METHODS USED FOR DIFFERENT TESTING FUNCTIONS
}

\author{
Pavel Raska ${ }^{\mathrm{a}}$, Zdenek Ulrych ${ }^{\mathrm{b}}$ \\ a Department of Industrial Engineering - FME, University of West Bohemia, \\ Univerzitni 22, Pilsen 306 14, Czech Republic \\ b Department of Industrial Engineering - FME, University of West Bohemia, \\ Univerzitni 22, Pilsen 306 14, Czech Republic
}

\begin{abstract}
The paper deals with testing and evaluation of selected modified heuristic optimization methods - Random Search, Downhill Simplex, Hill Climbing, Tabu Search, Local Search, Simulated Annealing, Evolution Strategy; Differential Evolution and Self Organizing Migrating Algorithm. The optimization methods were tested on four standard testing functions - the domain of the function is a defined step for each axis - substitution of the simulation model input parameter (discrete) values of the discrete event simulation model. The paper is mainly focused on testing the SOMA optimization method because this method is derived from Differential Evolution. Differential Evolution was a successful optimization for different dimensional search spaces. We proposed different evaluation criteria. We also tested different settings of these optimization methods to analyse their behaviour considering the setup of the optimization method parameters.
\end{abstract}

Keyword: Simulation optimization; optimization methods; Self Organizing Migrating Algorithm; testing function; evaluation
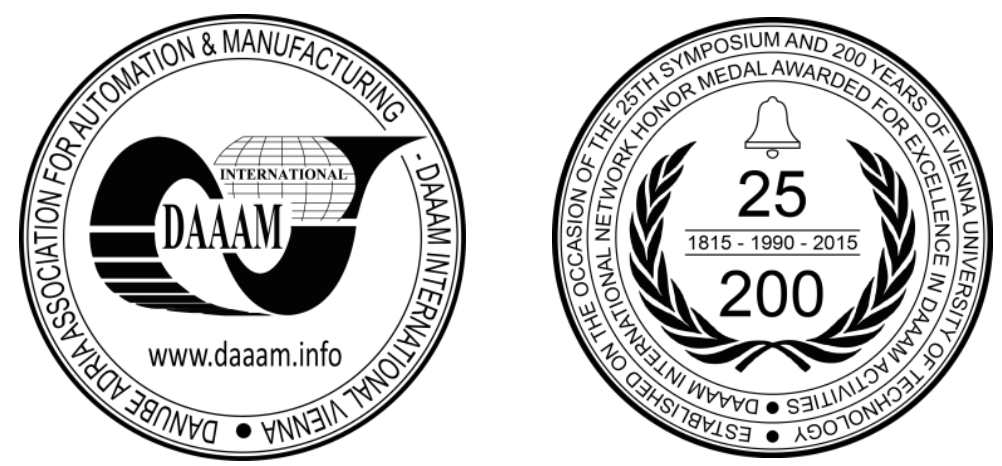

This Publication has to be referred as: Raska, P[avel] \& Ulrych, Z[denek] (2016). Comparison of a Modified SelfOrganizing Migrating Algorithm with other Selected Optimization Methods used for Different Testing Functions, Proceedings of the 26th DAAAM International Symposium, pp.0453-0461, B. Katalinic (Ed.), Published by DAAAM International, ISBN 978-3-902734-07-5, ISSN 1726-9679, Vienna, Austria DOI:10.2507/26th.daaam.proceedings.060 


\section{Introduction}

Simulation optimization can be defined as the process of finding suitable settings of decision variables of a simulation model according to specified restrictions to this model. In other words, we could say that simulation optimization helps us make better choices through the testing of various solutions to find a suitable setting of the simulated system under specified conditions. The solution has to be feasible regarding the modelled problem. Problems in the optimization of production processes are often NP hard. It is not possible to calculate all the possible variations to find the global optimum.

Much of today's simulation software used for modelling company production processes (e.g. Arena [1], Witness [2], PlantSimulation [3], etc.) uses its own integrated simulation optimizers. [4]

We have identified some optimization methods which are commonly used by simulation optimizers which are integrated in most recent simulation software. Selected methods are: Random Search, Downhill Simplex, Hill Climbing, Tabu Search, Local Search, Simulated Annealing [5], Evolution Strategy [6], Differential Evolution [7].

Some of these integrated optimizers are "Black boxes" trying to combine different methods in order to find appropriate solutions, and therefore the optimization method used for the solution cannot be identified. There are other problems such as: the user cannot set the parameters of the optimization method even when the user knows how to set these parameters; the modelled problem is similar to a previous modelled problem and the setting of optimization method parameters is known; the user cannot usually implement his own methods; the user cannot specify a termination criteria for the optimization run; it is not possible to create a knowledge database, etc.

On the basis of these problems and also to reflect the needs of research and the practical sphere we have developed our own simulation optimization application. This simulation optimizer can optimize the simulation models built in Arena and PlantSimulation simulation software. This application addresses the problems listed above - namely implementing modified optimization methods. The user can perform a detailed analysis of the simulation results.

Considering the time requirements of testing different settings of optimization methods and testing their efficiency of finding the optimum of discrete event simulation models [8], [9] we substitute the testing objective function of the simulation models with a different testing function - De Jong's, Rosenbrock's, Michalewicz's and Ackley's functions.

\section{Testing functions}

The domain of the function is defined and also a step is defined for each axis. This is a representation of substitution of the simulation model input parameter discrete values of the discrete event simulation model. The problem is to find suitable settings for the testing function input parameters (substitution of decision variables of the simulation model):

$$
\mathbf{X}=\left\lfloor x_{j}\right\rfloor \forall j: j=\{1,2, \ldots, n\}
$$

where $\mathbf{X}$ denotes the concrete setting of the input parameters; $x_{j}$ denotes the value of the $j$-th testing function input parameter; $n$ denotes the dimension of the search space. Each point (the concrete setting of the input parameters $\mathbf{X}$ ) of the search space $\widetilde{X}$ is a possible solution (candidate solution).

The search space in the case of Box Constraint can be formulated as follows:

$$
\tilde{X}=\prod_{j=1}^{n}\left[a_{j}, b_{j}\right] \forall j: j=\{1,2, \ldots, n\}
$$

where $a_{j}$ denotes the lower boundary of the $j$-th testing function input parameter; $b_{j}$ denotes the lower boundary of the $j$-th testing function input parameter. The candidate solution in the search space is evaluated by the objective function value $F(\mathbf{X})$ - the range commonly includes real numbers. This objective function represents the quality of candidate solution regarding the specified objective.

All testing functions representing the objective function $F(\mathbf{X})$ of a discrete event simulation model were minimized. It can be formulated as follows:

$$
\breve{\mathbf{X}}=\arg \min _{\mathbf{X} \in \tilde{X}} F(\mathbf{X})=\{\breve{\mathbf{X}} \in \tilde{X}: F(\breve{\mathbf{X}}) \leq F(\mathbf{X}) \forall \mathbf{X} \in \tilde{X}\}
$$

where $\breve{\mathbf{X}}$ denotes the global minimum of the objective function; $F(\mathbf{X})$ denotes the objective function value of the candidate solution; $\widetilde{X}$ denotes the Search space. [10] 


\subsection{De Jong's Function}

A convex and unimodal testing function. The function definition: [11]

$$
F(\mathbf{X})=\sum_{j=1}^{n} x_{j}^{2}, \forall x_{j}:\left(x_{j}-\left\lfloor\frac{x_{j}}{0.01}\right\rfloor \cdot 0.01=0\right) \wedge\left(-30 \leq x_{j} \leq 30\right), j=1: n, n \in\{2,8,10,20,30,40,50\}
$$

where $F(\mathbf{X})$ denotes the objective function; $j$ denotes index of control; $n$ denotes the dimension of the search space - the dimension of the search space is $2 ; 8 ; 10 ; 20 ; 30 ; 40 ; 50 ; x_{j}$ denotes the value of control - testing functions (except Michalewicz function) input parameters values range from -30 (lower boundary) to 30 (higher boundary). We defined the smallest step that can be performed by the optimization methods as 0.01 for each axis in the search space $\left(x_{j}\right.$ mod $0.01=0$ ). The testing function input parameters are not continuous. This resolution represents 36,012,001 possible points in a two dimensional search space. These points represent the combinations of testing function input parameters candidate solutions. The same resolution was used for a fifty dimensional search space $\left(8.1504 \times 10^{188}\right.$ possible candidate solutions).

\subsection{Rosenbrock's Function}

Rosenbrock's (Rosenbrock's valley, Rosenbrock's banana) function is a unimodal and non convex testing function. The function definition: [11]

$$
F(\mathbf{X})=\sum_{j=1}^{n-1} 100 \cdot\left(x_{j}^{2}-x_{j+1}\right)^{2}+\left(1-x_{j}\right)^{2}, \forall x_{j}:\left(x_{j}-\left\lfloor\frac{x_{j}}{0.01}\right\rfloor \cdot 0.01=0\right) \wedge\left(-30 \leq x_{j} \leq 30\right), j=1: n, n \in\{2,8,10,20,30,40,50\}
$$

\subsection{Michalewicz Function}

The Michalewicz function is a multimodal test function ( $\mathrm{n}$ ! local optima). The parameter $m$ defines the "steepness" of the valleys or edges. Larger $m$ leads to a more difficult search. For very large $m$ the function behaves like a needle in a haystack (the function values for points in the space outside the narrow peaks give very little information on the location of the global optimum). [11]

$$
F(\mathbf{X})=-\sum_{j=1}^{n} \sin \left(x_{j}\right) \cdot\left(\sin \left(\frac{j \cdot x_{j}^{2}}{\pi}\right)\right)^{2 \cdot m}, \forall x_{j}:\left(x_{j}-\left\lfloor\frac{x_{j}}{0.01}\right\rfloor \cdot 0.01=0\right) \wedge\left(0 \leq x_{j} \leq \pi\right), j=1: n, n \in\{2,8,10,20,30,40,50\}
$$

We selected $m=5$ in our simulation model.

\subsection{Ackley's Functions}

Ackley's function is a multimodal test function. This function is a widely used testing function for premature convergence.

$$
F(\mathbf{X})=-20 \cdot \exp \left(-0.02 \cdot \sqrt{\frac{1}{n} \cdot \sum_{j=1}^{n} x_{j}^{2}}\right)-\exp \left(\frac{1}{n} \cdot \sum_{j=1}^{n} \cos 2 \cdot \pi \cdot x_{j}\right)+20+\exp (1), \forall x_{j}:\left(x_{j}-\left\lfloor\frac{x_{j}}{0.01}\right\rfloor \cdot 0.01=0\right) \wedge\left(-30 \leq x_{j} \leq 30\right), j=1: n, n \in\{2,8,10,20,30,40,50\}
$$

\section{Series of optimization experiments}

We tested optimization methods on four selected testing functions where the dimension of the search space was $2 ; 8 ; 10 ; 20 ; 30 ; 40 ; 50$. The same conditions had to be satisfied for each optimization method, e.g. the same termination criteria:

The optimization method could perform a maximum of $20,000 \times n$ (parameter $n$ denotes the dimension of the search space) simulation experiments to find the global optimum in the search space [6] or the termination criterion was met if the optimum was found - VTR (value to reach); the same search space where the optimization method can search for the global optimum, etc. Error! Reference source not found. shows the average optimization method success of finding optimum. We accept the found candidate solution if its testing (objective) function value from the objective function value of global optimum does not exceed $\varepsilon=0.001$ - suboptimum.

If the global optimum of the testing function is not known (e. g. Michalewicz testing function) the optimum is represented by the best found candidate solution of all performed simulation experiments performed on the testing function. 


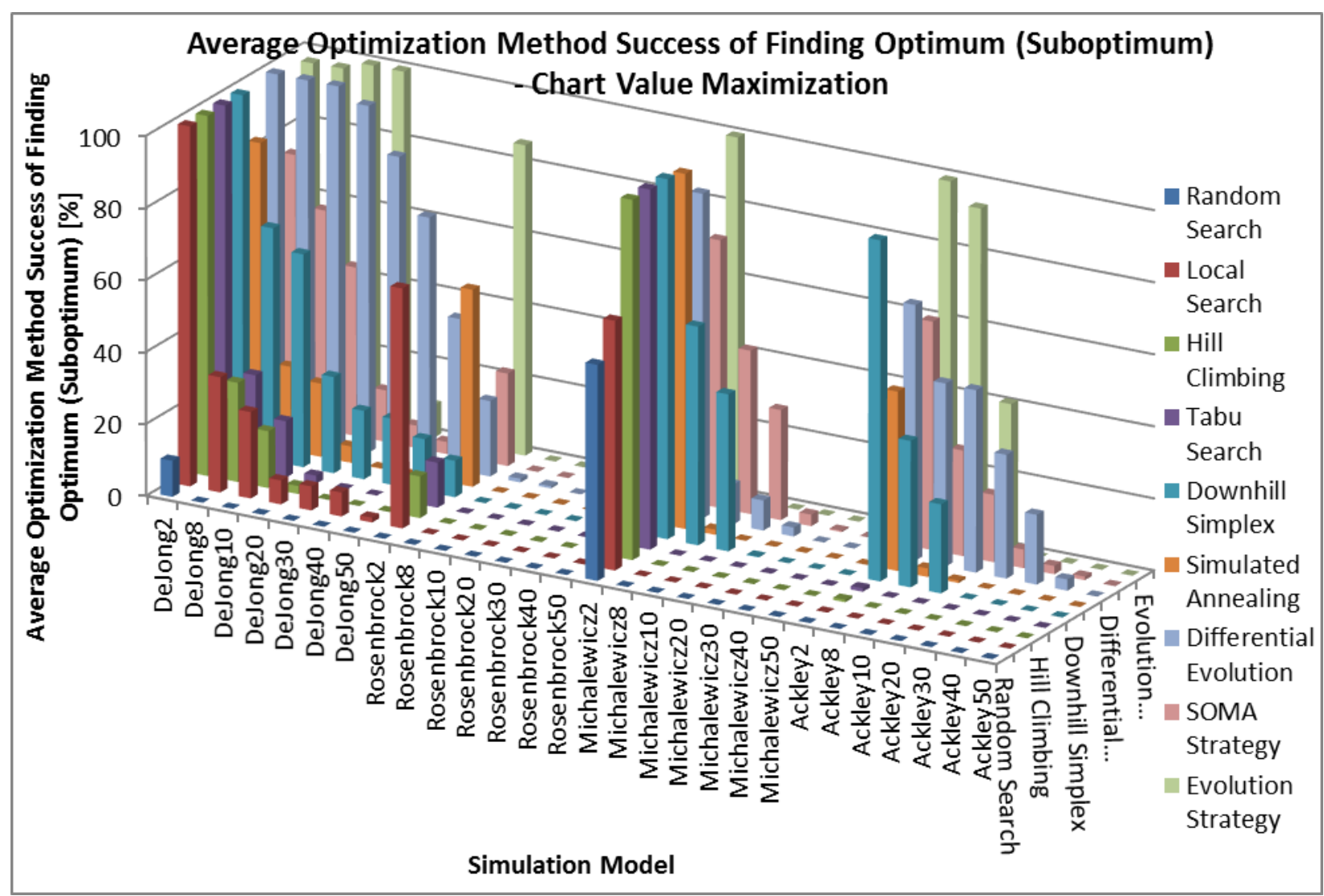

Fig. 1. Average Optimization Method Success of Finding Optimum (Suboptimum) - Chart Value Maximization - each series contains $20,000 \times \mathrm{n}$ simulation experiments; $\varepsilon=0.001$

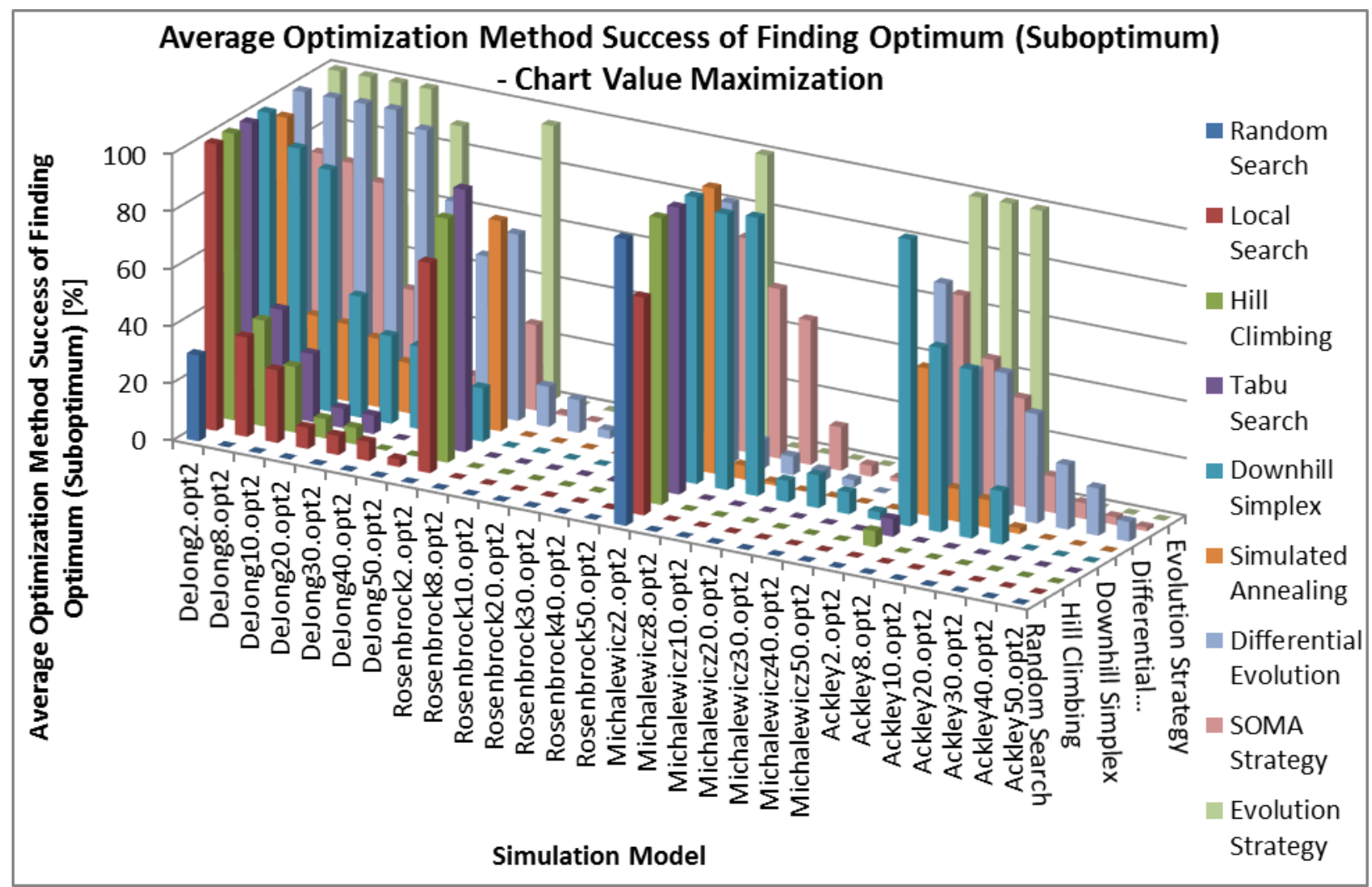

Fig. 2. Average Optimization Method Success of Finding Optimum (Suboptimum) - Chart Value Maximization - each series contains $100,000 \times \mathrm{n}$ simulation experiments; $\varepsilon=0.001$ 


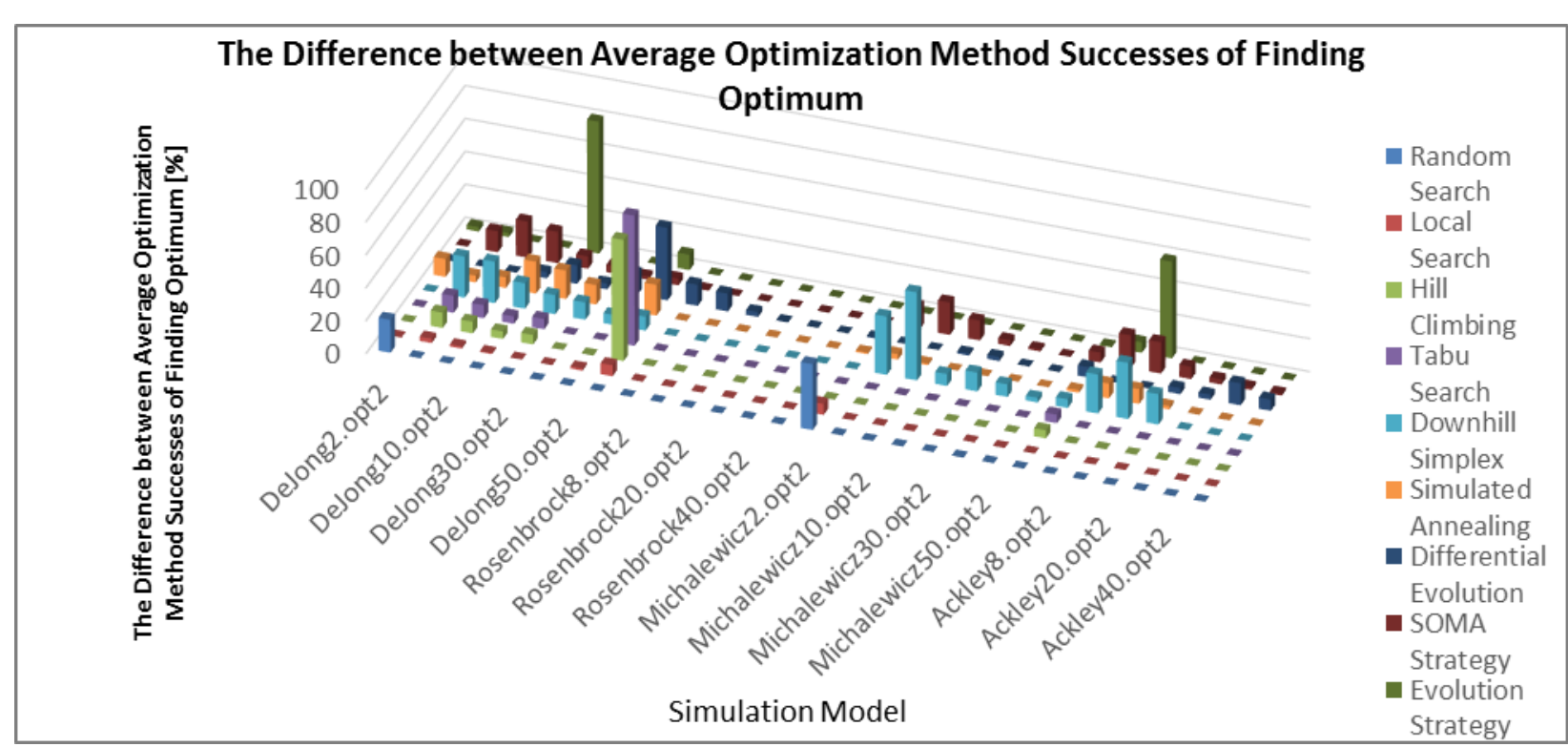

Fig. 3. The difference between average optimization method successes of series where a maximum of $20,000 \times n$ and a maximum of $100,000 \times \mathrm{n}$ simulation experiments; $\varepsilon=0.001$

In the next series of optimization experiments the optimization method could perform a maximum of $100,000 \times n$ simulation experiments (larger dimension of the search space) to find the global optimum in the search space or the termination criterion was met if the optimum was found - VTR - see Error! Reference source not found.

We performed simulation optimization experiments with different optimization method settings to find a suitable setting. If the optimization method has the same parameters as another optimization method, we set up both parameters with the same boundaries (same step, same lower and upper boundaries).

We compared the difference between the average optimization method successes of finding the optimum where the optimization method could perform a maximum of $20,000 \times n$ and a maximum of $100,000 \times n$ simulation experiments.

As we expected, the larger number of simulation experiments leads to improvement of finding the optimum by the optimization method. We averaged the differences between average optimization method successes of series where a maximum of $20,000 \times n$ and a maximum of $100,000 \times n$ simulation experiments where performed. Downhill Simplex methods were better able to find the global optimum with an average of 10 percent with the larger number of performed simulation experiments - see Error! Reference source not found.. Evolution Strategy increases its efficiency of finding the optimum by 80 percent on the De Jong 30 simulation model and by 58 percent on the Ackley 10 simulation model if a maximum of $100,000 \times n$ simulation experiments were performed.

\section{SOMA}

SOMA is based on the self-organizing behaviour of groups of individuals in a "social environment". It can also be classified as an evolutionary algorithm, despite the fact that no new generations of individuals are created during the search. Only the positions of the individuals in the search space are changed during a generation, called a "migration loop". Individuals are generated at random according to what is called the "specimen of the individual" principle. The specimen is in a vector, which comprises an exact definition of all these parameters that together led to the creation of such individuals, including the appropriate constraints of the given parameters. SOMA is not based on the philosophy of evolution (two parents create one new individual - the offspring), but on the behaviour of a social group of individuals. [12]

SOMA optimization method is derived from Differential Evolution. There exists different modifications of the Differential Evolution e. g. [13], [14].

\subsection{SOMA Parameters}

We performed many optimization experiments with a specific optimization method setting to reduce the influence of the random behaviour of the optimization method. We performed simulation optimization experiments with different optimization method settings to find a suitable setting. We set up optimization method parameters with the same boundaries (same step, same lower and upper boundaries) for each model.

Furthermore, we selected only those series of the optimization where the value of the number of found optimum or suboptimum equals the best found value of this criterion from all tested series. We found that at least one series had a $100 \%$ success for finding the global optimum from all the performed series of the optimization method. 
We subsequently counted the number of series with a $100 \%$ success rate with a specific setting of the optimization method parameter for each simulation model. This number was divided by the total number of series performed with the selected parameter value for a specific model, regardless of the success of a particular series. This calculated value expresses the percentage of series success. A series represents the specific parameter value of the optimization method.

We created a bar graph which expresses the success of a specific setting of the optimization method parameter. The height of the bar expresses the effect of the optimization parameter value on the optimization method success of finding the optimum. If these bars of the optimization method parameter are the same height we can say the optimization methods have little propensity to bad tuning of this parameter. [15]

\subsubsection{Mass}

The Mass parameter denotes how far the currently selected individual (candidate solution) stops from the leader individual (if the Mass = 1 then the currently selected individual stops at the position of the leader, if the Mass $=2$ then the currently selected individual stops behind the position of the leader, which equals the distance of the initial position of the currently selected individual and the position of the leader). If the Mass $<1$ then the currently selected individual stops in front of the leader which leads to degradation of the migration process (the algorithm finds only local extremes). Hence it is recommended to use Mass $>1$. It is also recommended to use the following lower and upper boundary of the parameter Mass $\in[1.1,3]$. [12]

The following chart (see Error! Reference source not found.) shows the percentage of absolutely successful series compared to all performed series with different settings of the Mass parameter (each series contains $100,000 \times n$ simulation experiments and $\varepsilon=0.001$ ). We found that the Mass parameter is not very sensitive to bad tuning parameters in our tested simulation models.

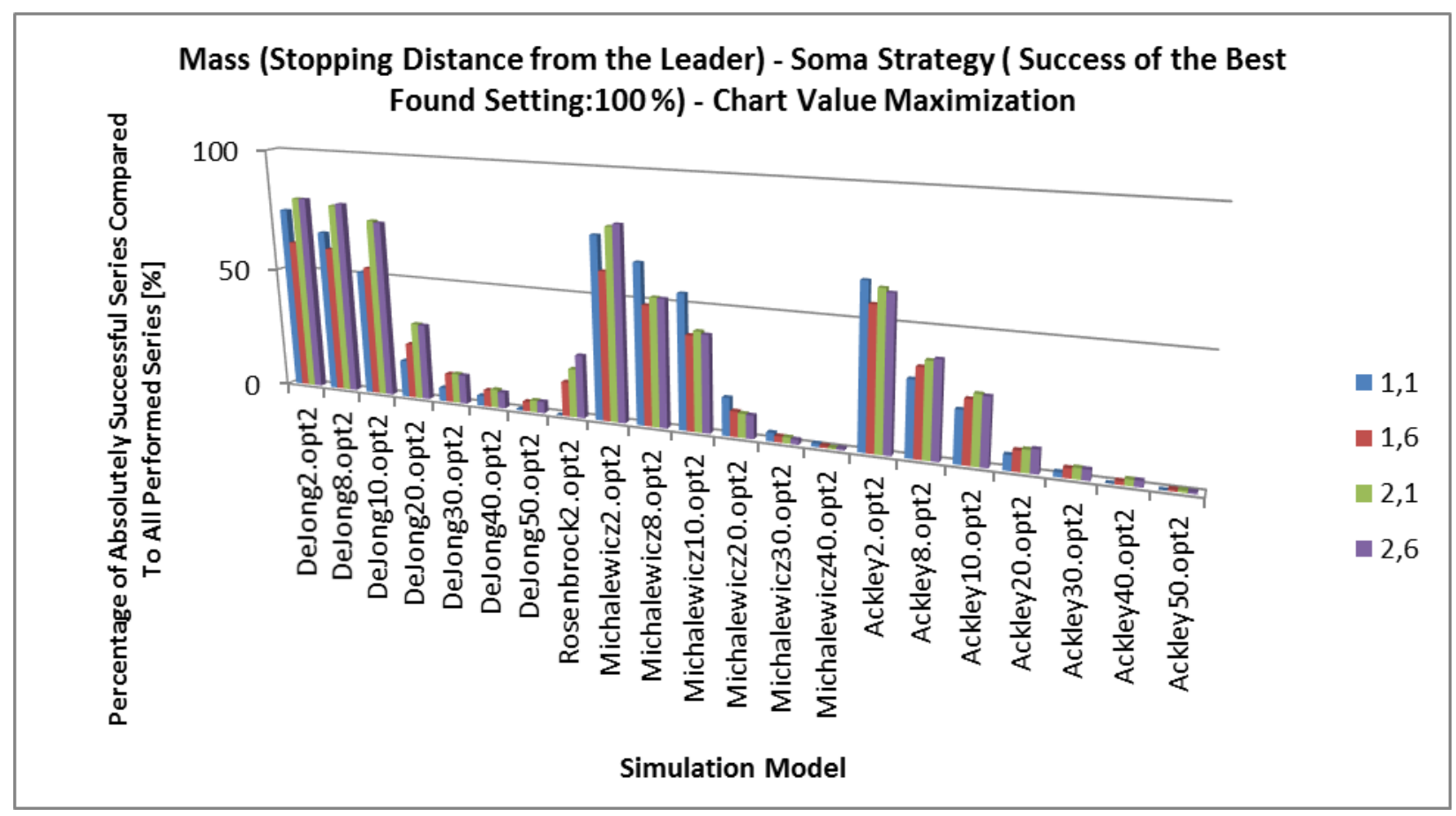

Fig. 4. Mass - Percentage of Absolutely Successful Series Compared to All Performed Series [\%] - Chart Value Maximization - each series contains $100,000 \times \mathrm{n}$ simulation experiments; $\varepsilon=0.001$

\subsubsection{Step}

The Step parameter denotes the resolution of mapping the path of the currently selected individual. It is possible to use a larger value for this parameter to accelerate the searching of the algorithm if the objective function is unimodal (convex function, few local extremes, etc.). If the objective function landscape is not known it is recommended to use a low value for this parameter. The search space will be scanned in more detail and this increases the probability of finding the global extreme. It is also important to set the Step parameter in a way that the distance of the currently selected individual and the leader is not an integer multiple of this parameter (the diversity of the population is reduced because each individual could be pulled to the leader and the process of searching for the optimum could stop in a local extreme). Hence it is recommended to use Step $=0.11$ instead of Step $=0.1$. The setting of e.g. Step $=0.11$ also rapidly increases the effectiveness of SOMA Strategy All To All. [12] 
The previous chart (see Error! Reference source not found.) shows the percentage of absolutely successful series compared to all performed series with different settings of the Step parameter. We can see that it is better to use a lower value for this parameter.

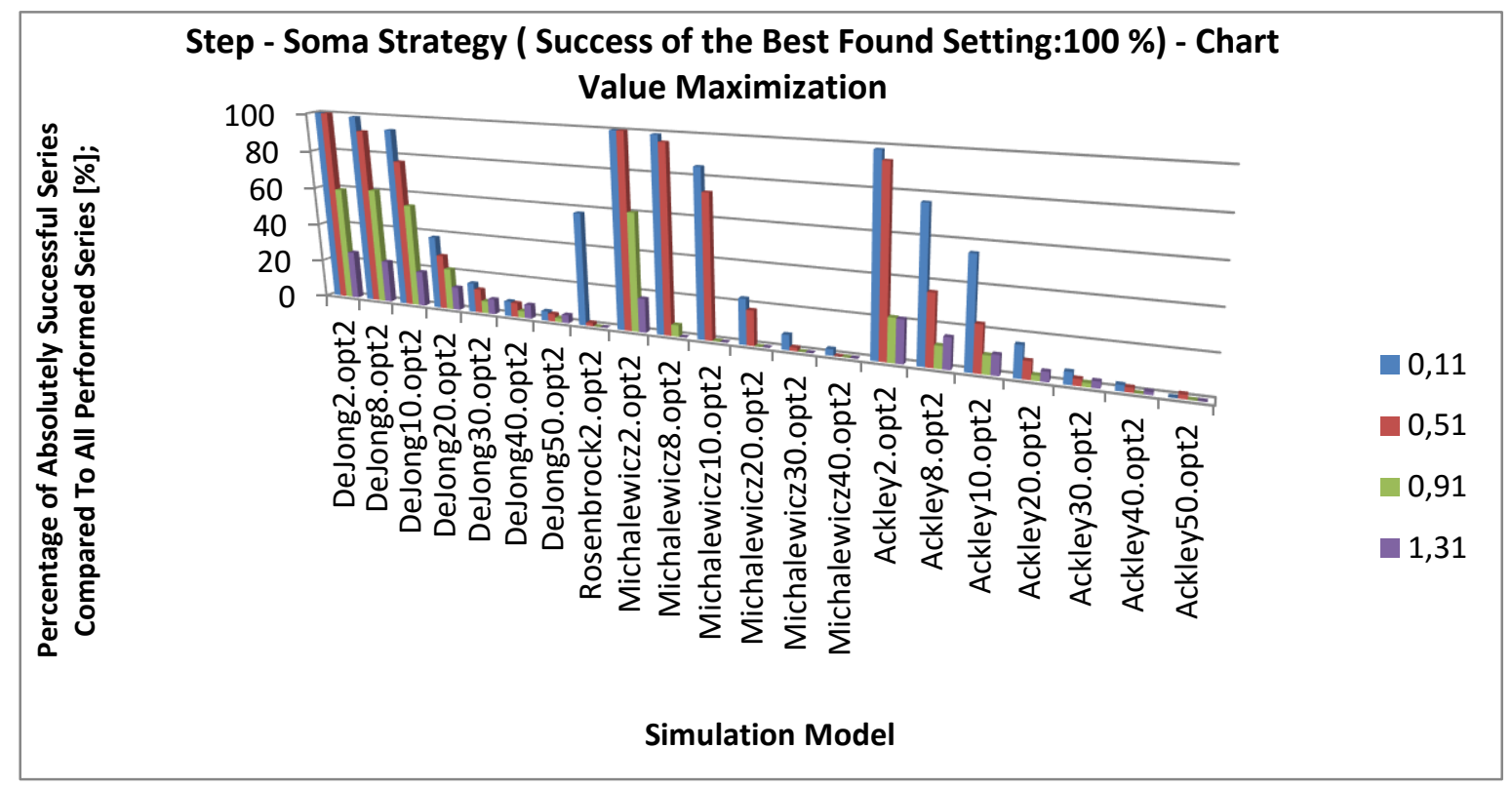

Fig. 5. Step - Percentage of Absolutely Successful Series Compared to All Performed Series [\%] - Chart Value Maximization - each series contains $100,000 \times \mathrm{n}$ simulation experiments; $\varepsilon=0.001$

\subsubsection{Perturbation}

PRT parameter denotes the perturbation. The Perturbation vector contains the information whether the movement of the currently selected individual toward the leader should be performed. It is one of the most important parameters of this optimization method and it is very sensitive. It is recommended to use $P R T=0.1$. If the value of this parameter increases, then the convergence of the SOMA algorithm to local extremes also rapidly increases. It is possible to set this parameter to PRT $\in[0.7,1.0]$ if many individuals are generated and if in the dimension of the search space the objective function is low. If $P R T=1$ then the stochastic part of behavior of SOMA is canceled and the algorithm behaves according to deterministic rules (local optimization of the multimodal objective function). [12]

The following chart (see Error! Reference source not found.) shows the percentage of absolutely successful series compared to all performed series with different settings of the Perturbation parameter. We can see that it is better to use a lower value of this parameter.

\subsubsection{NP Parameter}

This control parameter denotes how many individuals are generated in a population. If this parameter is set to $N P=2$ the SOMA algorithm behaves like a traditional deterministic method.

Generally if $n$ (where $n$ denotes the dimension of the search space) is a higher number, then this parameter can be set to $N P=[0.2,0.5] \times n$. If the objective function landscape is simple we can use a lower number of generated individuals. If the objective function is complicated we can set this parameter $N P=n$. It is recommended to use $N P \geq$ 10. [12]

The previous chart (see Error! Reference source not found.) shows the percentage of absolutely successful series compared to all performed series with different settings of $N P$ parameter. We performed series where the number of individuals in a population is equal to a multiple of the dimension of the search space. We found that $N P$ parameter is not very sensitive to bad tuning parameters in the case of our tested simulation models (the heights of the bars are nearly the same).

\subsubsection{Migrations}

This parameter is equivalent to the "Generation" parameter used in other evolutionary algorithms. This parameter denotes the number of population regenerations. [12] 


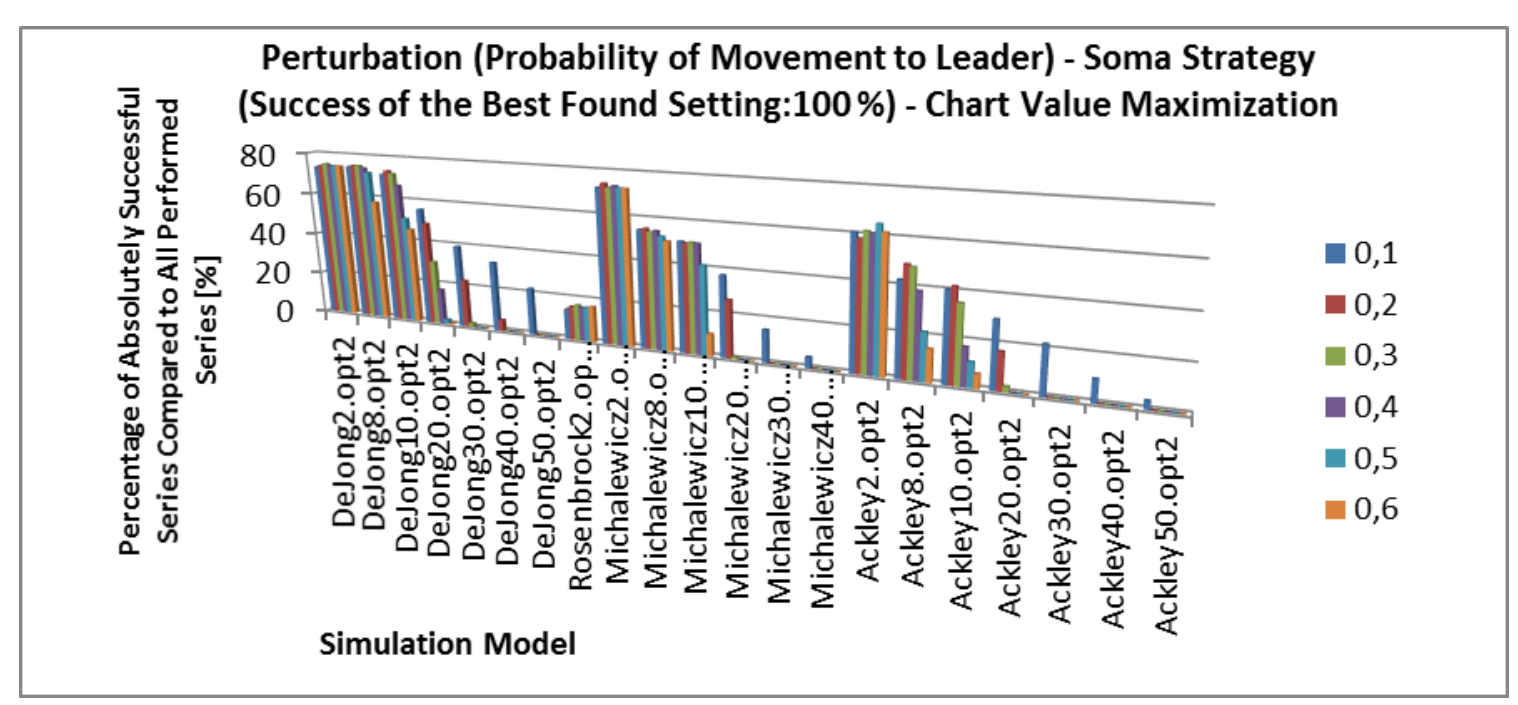

Fig. 6. Perturbation - Percentage of Absolutely Successful Series Compared to All Performed Series [\%] - Chart Value Maximization - each series contains $100,000 \times \mathrm{n}$ simulation experiments; $\varepsilon=0.001$

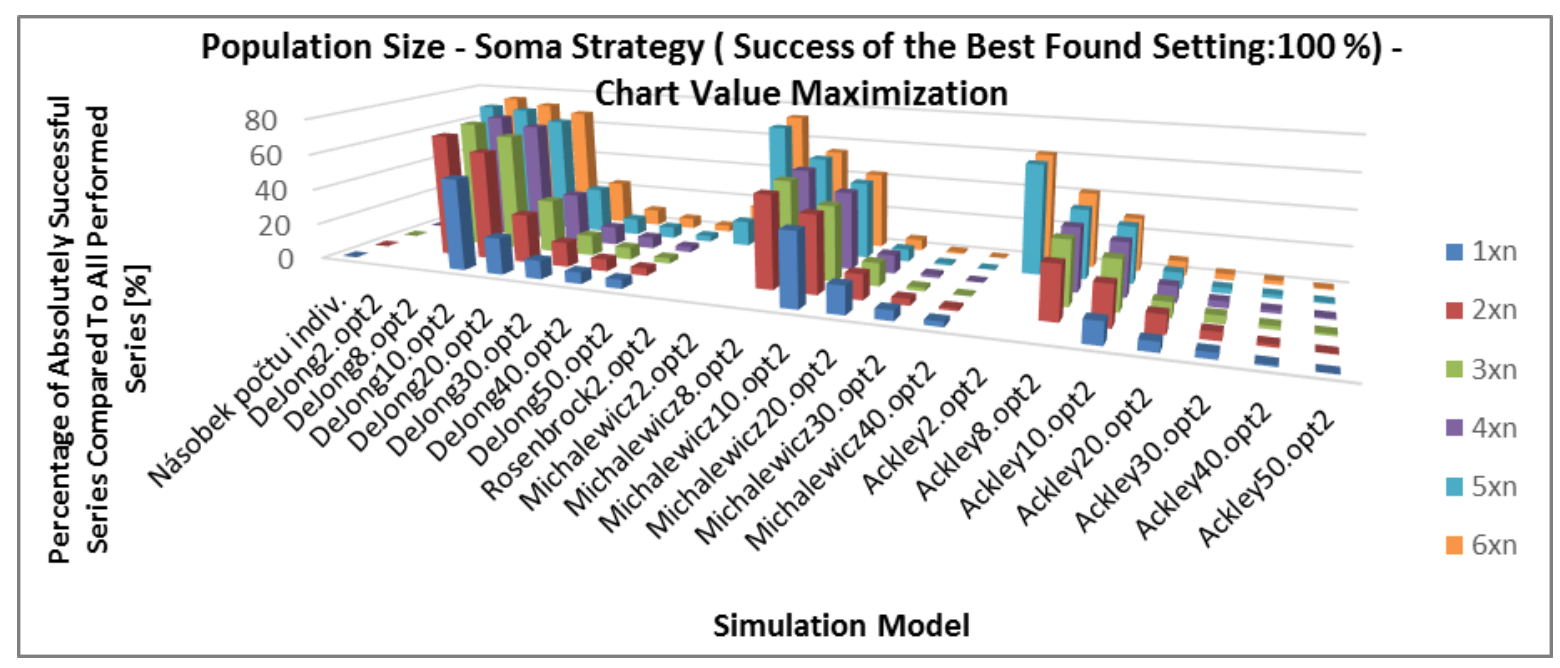

Fig. 7. Number of Individuals in Population - Percentage of Absolutely Successful Series Compared to All Performed Series [\%] - Chart Value Maximization - each series contains 100,000 ×n simulation experiments; $\varepsilon=0.001$

\section{Conclusion}

The goal of our research is to compare selected modified heuristic optimization methods - Random Search, Downhill Simplex, Hill Climbing, Tabu Search, Local Search, Simulated Annealing, Evolution Strategy; Differential Evolution and Self Organizing Migrating Algorithm. Optimization methods were used to search for the global optimum of the testing functions. We accept the found candidate solution if its testing (objective) function value from the objective function value of global optimum does not exceed $\varepsilon=0.001$. If the global optimum of the testing function is not known (e. g. Michalewicz testing function) the optimum is represented by the best found candidate solution of all the simulation experiments performed on the testing function.

We compared the difference between average optimization method successes of finding the optimum where the optimization method could perform a maximum of $20,000 \times n$ and a maximum of $100,000 \times n$ simulation experiments. As we expected, the larger number of simulation experiments leads to improvement of finding the optimum by the optimization method - namely in the case of Downhill Simplex.

We found the following conclusions regarding to performed simulation experiments. Evolution and SOMA Strategy methods were better able to find the global optimum if a larger number of simulation experiments was performed. The success of these selected heuristic optimization methods depends on the objective function landscape. Very good results were achieved with the Differential Evolution optimization method despite the larger number of dimensions of the search space. We also tested the SOMA optimization method because this method is derived from Differential Evolution. This method was successful due to the Differential Evolution only for the Michalewicz testing function. We performed simulation optimization experiments with SOMA optimization method settings to find a suitable setting and to analyse the behaviour of optimization methods considering the setup of the optimization method parameters. We also compared different strategies of individual movements -All To One, All To All, All To All 
Adaptive Random, All To One Random. We can say that the first strategy - All To One - should be preferred for all testing functions. simulation models.

We would like to test other optimization methods behaviours and optimization parameters setting on different

\section{Acknowledgements}

This paper was created with the subsidy of the project SGS-2015-065 "The development of parameters of a sustainable manufacturing system" carried out with the support of the Internal Grant Agency of the University of West Bohemia. The paper uses the knowledge and the results of the project CZ.1.07/2.3.00/09.0163 carried out with the support of ESF and State budget of the Czech Republic.

\section{References}

[1] Arena, OptQuest for Arena, Rockwell Automation, 2015. Available: http://www.arenasimulation.com. Accessed: 2015-07-03.

[2] WITNESS Optimizer, Lanner, Available: http://www.lanner.com/en/media/witness/optimiser.cfm.Accesed: 2015 07-10.

[3] Siemens, Plant Simulation, Siemens, 2015, Available: http://www.plm.automation.siemens.com/cz_cz/products/tecnomatix/plant_design/plant_simulation.shtml. Accessed: 2015-07-03.

[4] OR/MS, OR/MS TODAY, INFORMS - Institute for Operations Research and the Management Sciences, October 2015. Available: http://www.orms-today.org/surveys/Simulation/Simulation6.html. Accessed: 2015-10-01.

[5] A. J. Monticelli, R. Romero, E. N. Asada, Fundamentals of Simulated Annealing, in: Modern Heuristic Optimization Techniques, IEE Press ed., M. E. El-Hawary, Ed., New Jersey, John Wiley \& Sons, 2008, pp. 101120.

[6] J. Tvrdik, Stochastic Algorithms for Global Optimization (in Czech language: Stochastické algoritmy pro globální optimalizaci), University of Ostrava, 2010. Available: http://www1.osu.cz/ tvrdik/wpcontent/uploads/STAGO_10.pdf. Accessed: 2014-01-05.

[7] K. P. Wong, Z. Y. Dong, Differential Evolution, in: Modern Heuristic Optimization Techniques, M. E. El-Hawary, Editor, New Jersey, John Wiley \& Sons, 2008, pp. 171-186.

[8] P. Raska, Z. Ulrych, Testing optimization methods on discrete event simulation models and testing functions, Procedia Engineering, Vol. 69, pp. 768-777, 2014.

[9] P. Raska, Z. Ulrych, Comparison of Optimization Methods Tested On Testing Functions and Discrete Event Simulation Models, International Journal of Simulation and Process Modelling, 2015

[10] P. Raska, Z. Ulrych, Simulation Optimizer and Optimization Methods Testing On Discrete Event Simulations Models and Testing Functions, in: Proceedings of the European Modeling and Simulation Symposium, ATHENS, GREECE, 2013.

[11] H. Pohlheim, GEATbx: Example Functions, 2006]. Available: http://www.geatbx.com/docu/fcnindex01.html\#P204_10395. Accessed: 2011-11-20.

[12] I. Zelinka, SOMA - Self-Organizing Migrating Algorithm, New Optimization Techniques in Engineering Studies in Fuzziness and Soft Computing, Vol. 141, Berlin, Springer Berlin Heidelberg, pp. 167-217, 2004

[13] L. W. Vincent, S. G. Ponnambalam, A Differential Evolution-Based Algorithm to Schedule Flexible Assembly Lines, in: IEEE TRANSACTIONS ON AUTOMATION SCIENCE AND ENGINEERING, Vol. 10, No. 4, 2013, pp. $1161-1165$.

[14] Y. L. Li, Z. H. Zhan, Y. J. Gong, W. N. Chen, J. Zhang, Y. Li, Differential Evolution with an Evolution Path: A DEEP Evolutionary Algorithm, in: IEEE TRANSACTIONS ON CYBERNETICS, Vol. 45, No. 9, 2015, pp. 17981810.

[15] P. Raska, Z. Ulrych, Comparison of Modified Downhill Simplex and Differential Evolution with other Selected Optimization Methods used for Discrete Event Simulation Models, Procedia Engineering, Vol. 100, pp. 807-815, 2015. 